\title{
Plasma hyaluronan, hyaluronidase activity and endogenous hyaluronidase inhibition in sepsis: an experimental and clinical cohort study
}

\author{
Jaap van der Heijden ${ }^{1 *}\left(\mathbb{D}\right.$, Constantinos Kolliopoulos ${ }^{2}$, Paul Skorup ${ }^{3}$, Marko Sallisalmi ${ }^{4}$, Paraskevi Heldin², \\ Michael Hultström ${ }^{1,5}$ and Jyrki Tenhunen ${ }^{1}$
}

\section{*Correspondence:}

jaap.vanderheijden@surgsci. uu.se

${ }^{1}$ Department of Surgical Sciences, Anesthesiology and Intensive Care, Uppsala University, 75185 Uppsala, Sweden

Full list of author information is available at the end of the article

\begin{abstract}
Background: Plasma hyaluronan concentrations are increased during sepsis but underlying mechanisms leading to high plasma hyaluronan concentration are poorly understood. In this study we evaluate the roles of plasma hyaluronan, effective plasma hyaluronidase (HYAL) activity and its endogenous plasma inhibition in clinical and experimental sepsis. We specifically hypothesized that plasma HYAL acts as endothelial glycocalyx shedding enzyme, sheddase.

Methods: Plasma hyaluronan, effective HYAL activity and HYAL inhibition were measured in healthy volunteers $(n=20)$, in patients with septic shock $(n=17$, day 1 and day 4), in patients with acute pancreatitis ( $n=7$, day 1 and day 4 ) and in anesthetized and mechanically ventilated pigs $(n=16)$. Sixteen pigs were allocated (unblinded, open label) into three groups: Sepsis-1 with infusion of live Escherichia coli (E. coli) $1 \times 10^{8} \mathrm{CFU} / \mathrm{h}$ of $12 \mathrm{~h}(n=5)$, Sepsis- 2 with infusion of E. coli $1 \times 10^{8} \mathrm{CFU} / \mathrm{h}$ of $6 \mathrm{~h}$ followed by $1 \times 10^{9} \mathrm{CFU} / \mathrm{h}$ of the remaining $6 \mathrm{~h}(n=5)$ or Control with no $E$. coli infusion $(n=6)$.
\end{abstract}

Results: In experimental E. coli porcine sepsis and in time controls, plasma hyaluronan increases with concomitant decrease in effective plasma HYAL activity and increase of endogenous HYAL inhibition. Plasma hyaluronan increased in patients with septic shock but not in acute pancreatitis. Effective plasma HYAL was lower in septic shock and acute pancreatitis as compared to healthy volunteers, while plasma HYAL inhibition was only increased in septic shock.

Conclusion: Elevated plasma hyaluronan levels coincided with a concomitant decrease in effective plasma HYAL activity and increase of endogenous plasma HYAL inhibition both in experimental and clinical sepsis. In acute pancreatitis, effective plasma HYAL activity was decreased which was not associated with increased plasma hyaluronan concentrations or endogenous HYAL inhibition. The results suggest that plasma HYAL does not act as sheddase in sepsis or pancreatitis.

Keywords: Hyaluronan, Hyaluronidase, Hyaluronidase inhibitor, Sepsis, Pancreatitis, Glycocalyx, Sheddase author(s) and the source, provide a link to the Creative Commons licence, and indicate if changes were made. The images or other third party material in this article are included in the article's Creative Commons licence, unless indicated otherwise in a credit line to the material. If material is not included in the article's Creative Commons licence and your intended use is not permitted by statutory regulation or exceeds the permitted use, you will need to obtain permission directly from the copyright holder. To view a copy of this licence, visit http:// creativecommons.org/licenses/by/4.0/. 


\section{Introduction}

Sepsis is a life-threatening syndrome with high incidence, morbidity and mortality [1]. The pathophysiology is complex and still largely unknown making sepsis a challenge for clinicians and researchers alike. Current therapeutic options are limited and treatment is mainly based on early detection, broad-spectrum antibiotics, fluid resuscitation, vasopressors and organ support [2].

Plasma concentrations of hyaluronan are up to twenty times higher in septic patients compared with healthy individuals [3] and correlate with organ dysfunction [4] and mortality $[5,6]$. The exact mechanisms leading to or any potential consequences of high plasma hyaluronan are poorly understood. Hyaluronan is a long non-sulfated polysaccharide composed of repeated disaccharide units of glucuronic acid and $\mathrm{N}$-acetylglucosamine with a molecular weight that extends up to several million Daltons. The functional effects of hyaluronan are size dependent; high molecular weight hyaluronan (HMW-HA $>500 \mathrm{kDa}$ ) is anti-inflammatory, whereas fragmented hyaluronan is proinflammatory and pro-angiogenic [7, 8]. HMW-HA is very hygroscopic and one gram can bind up to $6 \mathrm{l}$ of water [9]. An average human body contains $15 \mathrm{~g}$ of hyaluronan, which is unevenly distributed and mostly found in the skin (56\%), skeleton and connective tissues (27\%). Up to a third of the total hyaluronan content is turned-over each day, which means that small changes in either the production, transport or degradation can have major impact in tissue fluid balance and edema formation [10].

The metabolism of hyaluronan in humans is mediated through synthetic enzymes (HAS1-3), catabolic hyaluronidases (HYAL) and endogenous HYAL inhibitors [11-13]. Hyaluronan is synthesized at the plasma membrane and exported into the extracellular matrix. Hyaluronan is degraded in tissue by HYAL and reactive oxygen species or transported via the lymphatic system and degraded by HYAL in lymph nodes. Excess of hyaluronan enters the circulation and is removed mainly by the liver through HYAL. The HYAL family was first reported as "Spreading Factor" due to the ability to break down hyaluronan in the extracellular matrix and, therefore, increasing the invasiveness of bacteria and venoms $[14,15]$. Little is known about endogenous HYAL inhibitors but a predominant HYAL inhibitor is hypothetically a $120 \mathrm{kDa}$ member of the Inter-alphaInhibitor protein (I $\alpha \mathrm{Ip})$ [16].

Several hypotheses have been proposed to explain increased plasma hyaluronan concentrations in sepsis. First, viral and bacterial infection and inflammatory mediators associated with sepsis (TNF- $\alpha$, interleukin-1 and growth factors) increase hyaluronan production $[17,18]$. Second, increased lymphatic outflow mobilizes more hyaluronan from the interstitium in experimental sepsis [19]. Decreased HYAL activity in either tissue, lymphatics and liver could lead to increased plasma hyaluronan concentration [17]. Finally, the endothelial surface layer, or glycocalyx, is damaged during sepsis. The glycocalyx is rich in hyaluronan and sepsis-induced shredding of this layer could contribute to increased plasma hyaluronan concentration [20]. Injection of HYAL has been shown to induce degradation of glycocalyx [21] and circulating HYAL is, therefore, considered as a potential endogenous sheddase [22, 23]. Any potential role of HYAL and its' endogenous plasma inhibitors in sepsis is unknown.

The aim of this study was to investigate how plasma HYAL activity and plasma HYAL inhibition correlates with hyaluronan during systemic inflammation in a porcine E. coli 
sepsis model and in clinical human blood samples in sepsis or non-infectious inflammation in pancreatitis. We specifically hypothesized that plasma HYAL acts as an endothelial glycocalyx shedding enzyme.

\section{Materials and methods}

\section{Preclinical experimental study}

\section{Anaesthesia and instrumentation}

Sixteen healthy Swedish landrace-breed piglets $(24.6 \pm 1.5 \mathrm{~kg})$ were used in the study. The animals had free access to water and food until transportation to the facility. All animals were given $50 \mathrm{mg}$ xylazine intramuscularly before transport to minimize stress. At arrival, the animals were weighed and tiletamine-zolazepam $6 \mathrm{mg} / \mathrm{kg}$ and xylazine $2.2 \mathrm{mg} / \mathrm{kg}$ were given intramuscularly. The ear vein was cannulated and $20 \mathrm{mg}$ of morphine was given. Anaesthesia was maintained using continuous intravenous infusion of sodium-pentobarbital $8 \mathrm{mg} / \mathrm{kg} / \mathrm{h}$, morphine $0.48 \mathrm{mg} / \mathrm{kg} / \mathrm{h}$ and rocuronium bromide $1.5 \mathrm{mg} / \mathrm{kg} / \mathrm{h}$. A preload bolus of $20 \mathrm{ml} / \mathrm{kg}$ Ringer's Acetate was given followed by a continuous infusion of $2 \mathrm{ml} / \mathrm{kg} / \mathrm{h}$ glucose $25 \mathrm{mg} / \mathrm{ml}$. The total fluid administration, excluding interventional boluses, was $4.26 \mathrm{ml} / \mathrm{kg} / \mathrm{h}$. The airway was secured by tracheostomy followed by volume-controlled ventilation with tidal volumes of $6 \mathrm{ml} / \mathrm{kg}$, inspiratoryto-expiratory ratio of 1:2, fractional inspired oxygen $\left(\mathrm{FiO}_{2}\right) 0.30$, positive end-expiratory pressure (PEEP) $5 \mathrm{cmH}_{2} \mathrm{O}$, and respiratory rate $25 / \mathrm{min}$.

A urine catheter was placed through a vesicostomy to monitor hourly urine production. The right external jugular vein was catheterized with a pulmonary arterial catheter and central venous catheter to monitor cardiac index (CI), mean pulmonary arterial pressure (MPAP) and pulmonary capillary wedge pressure (PCWP). The right cervical artery was catheterized in to measure blood pressure and to collect blood samples. A 45-60 min of stabilization was allowed to achieve normoventilation with an arterial partial pressure of carbon dioxide $\left(\mathrm{PaCO}_{2}\right)$ between 35 and $45 \mathrm{mmHg}(5.0$ and $5.5 \mathrm{kPa})$ by adjusting the respiratory rate. Body temperature was maintained between 38.0 and $39.5^{\circ} \mathrm{C}$ using a warming mattress and blankets.

\section{Induction of sepsis}

Sepsis was induced by infusion of live Escherichia coli (E. coli, strain B09-11822) as previously reported [24]. We chose to use E. coli, because it does not express HYAL [25]. The bacterial infusion was replaced every second hour to keep the bacteria in a logarithmic growth phase. Sixteen animals were allocated into three groups (unblinded, open label): Sepsis- 1 ( $n=5$, infusion of E. coli $1 \times 10^{8} \mathrm{CFU} / \mathrm{h}$ during $12 \mathrm{~h}$ ), Sepsis-2 $(n=5$, infusion of $E$. coli $1 \times 10^{8} \mathrm{CFU} / \mathrm{h}$ during $6 \mathrm{~h}$ followed by infusion of $10 \times 10^{8} \mathrm{CFU} / \mathrm{h}$ during the remaining $6 \mathrm{~h}$ ) and Control ( $n=6$, infusion of equal amount of $0.9 \%$ saline). We used daily two parallel animals consecutively allocated to each group.

Respiratory and circulatory parameters were followed continuously and recorded every hour. Blood samples (EDTA), arterial blood gas and mixed venous blood gas $\left(\mathrm{SvO}_{2}\right)$ were taken hourly. Urine samples and blood samples for bacterial count were collected every $3 \mathrm{~h}$. Total blood loss was estimated to be less than $10 \%$ of the circulating blood volume (Additional file 1: Fig. S1, time-line of preclinical experimental design). 
Respiratory and circulatory interventions were protocolized according to current intensive care practice (excluding antibiotics treatment) and summarized in Additional file 2: Table S1. At the experimental endpoint the animals were killed by a direct injection of potassium chloride in the central venous line while under anaesthesia.

\section{Clinical study}

Healthy volunteers $(n=20)$, patients with septic shock $(n=17)$ and acute alcoholic pancreatitis $(n=7)$ were enrolled from September 2008 to September 2009 at the Helsinki University Central Hospital. All data for patients with acute pancreatitis is previously unpublished, while the distribution of molecular weight for hyaluronan in plasma among healthy volunteers' and septic shock patients' were previously published [3]. Inclusion criteria for patients with septic shock were: age $\geq 18$ years, $\geq 2$ of SIRS criteria fulfilled, proven or highly suspected infection consistent with septic shock and norepinephrine therapy of at least $0.1 \mathrm{mcg} / \mathrm{kg} / \mathrm{min}$ in spite of adequate fluid resuscitation to maintain systemic arterial pressure (SAP) above $90 \mathrm{mmHg}$. Inclusion criteria for patients with acute pancreatitis were: age $\geq 18$ years, $\geq 2$ of SIRS criteria fulfilled, pancreatitis confirmed by a computer tomography in the absence of biliary stones and a history of excessive consumption of alcoholic beverages (ethanol). Blood samples were taken within $24 \mathrm{~h}$ (day 1 ) of admission to the ICU and $72 \mathrm{~h}$ (day 4 ) thereafter. Plasma samples were collected in K2-EDTA-tubes and centrifuged at $18{ }^{\circ} \mathrm{C}$ for $15 \mathrm{~min}(2500 \mathrm{~g})$ and stored at $-80^{\circ} \mathrm{C}$ for further analysis.

\section{Quantification of hyaluronan, effective HYAL activity and endogenous HYAL inhibition in plasma}

We quantified hyaluronan using an ELISA-like assay as described earlier [26]. An assay was adapted and optimized to measure effective HYAL activity [27] and the HYAL inhibition assay was based on the HYAL activity protocol with adjustments [16, 28, 29]. In detail, please see Additional file 3: text analysis.

\section{Statistics}

We chose to use a convenience sample size with minimal reasonable number of animals for the experimental study, since no prior data of plasma HYAL activity and plasma HYAL inhibition in septic pigs were available. Statistical analyses were preformed using IBM $^{\circledR}$ SPSS $^{\circledR}$ statistics version 23 (SPSS, Inc., Chicago, IL, USA). Normality of distribution of continuous variables was tested by Shapiro-Wilk test and visually assessed using histograms. When necessary, the Expectation Maximization method for missing data was applied. One-way ANOVA or Kruskal-Wallis test were used to compare the groups. Wilcoxon signed-rank test was used to compare groups over time. Two-way repeated measures ANOVA was used to compare differences within and between the groups over time. If relevant post hoc analysis was performed with the Bonferroni correction or Mann Whitney $U$ tests. Spearman's rank-order test was used for correlation analysis. For within-subjects correlation we used the Bland Altman method [30]. The statistical tests were used according to the distribution of the data. A value of $p<0.05$ was considered statistically significant. 


\section{Results}

\section{Porcine sepsis: bacterial infusion, respiratory and circulatory variables}

The bacterial load for all Sepsis-1 (T0-12) experiments and Sepsis-2 (T0-6) was $0.9 \times 10^{8} \mathrm{CFU} / \mathrm{h}$ (IQR 0.73-1.43) compared with $11.8 \times 10^{8} \mathrm{CFU} / \mathrm{h}$ (IQR 10.9-14.0) for Sepsis-2 (T6-12). No live bacteria were detectable in blood cultures in the control group (Additional file 1: Fig. S2). The groups were comparable at the baseline except for blood lactate being higher in Sepsis-1 group (Additional file 2: Table S2). Animals in the control group were stable in circulatory and respiratory parameters throughout the experiment. In both sepsis groups, one animal died between 6 and $7 \mathrm{~h}$ after infusion with live E. coli following circulatory and respiratory collapse despite the predetermined interventions. The 12-h sepsis experiments were characterized by decreased mean arterial pressure (MAP), $\mathrm{SvO}_{2}$ and $\mathrm{PaO}_{2} / \mathrm{FiO}_{2}$ ratio, increased arterial lactate and a transient increase in mean pulmonary arterial pressure (MPAP) as compared to baseline and controls (Fig. 1a-f). No differences were found between Sepsis-1 and Sepsis-2. The requirement for fluid resuscitation was comparable in Sepsis-1 and Sepsis-2 $(2.5 \mathrm{ml} / \mathrm{kg} / \mathrm{h}$ (IQR $0.6-7.6)$ and $3.8 \mathrm{ml} / \mathrm{kg} / \mathrm{h}$ (IQR 2.5-15.3, $p=0.175$ ). Vasopressor was needed to maintain MAP $\geq 60$ in both Sepsis-1 $(n=3 / 5)$ and Sepsis-2 $(n=3 / 5)$ but no difference in vasopressor load was found between the groups $(p=0.746)$.

\section{Porcine sepsis: hyaluronan, effective HYAL activity and HYAL inhibition in plasma}

Plasma hyaluronan concentration increased over time in all three groups and was higher in the Sepsis-2 group [158 ng/ml (IQR 147-509)] as compared to Control (117 ng/ml (IQR 80-143), $p=0.011$ ) and Sepsis-1 (56 ng/ml (IQR 50-142), $\mathrm{p}=0.047$ ) groups after $12 \mathrm{~h}$ (Fig. 2a). Effective plasma HYAL activity decreased over the length of the experiment comparably in all three groups and no difference was found at $12 \mathrm{~h}$ (Fig. 2b). Concomitantly, activity of endogenous plasma HYAL inhibition increased over time in Control and Sepsis-1 groups (Fig. 2c). The within-subject analyses for repeated

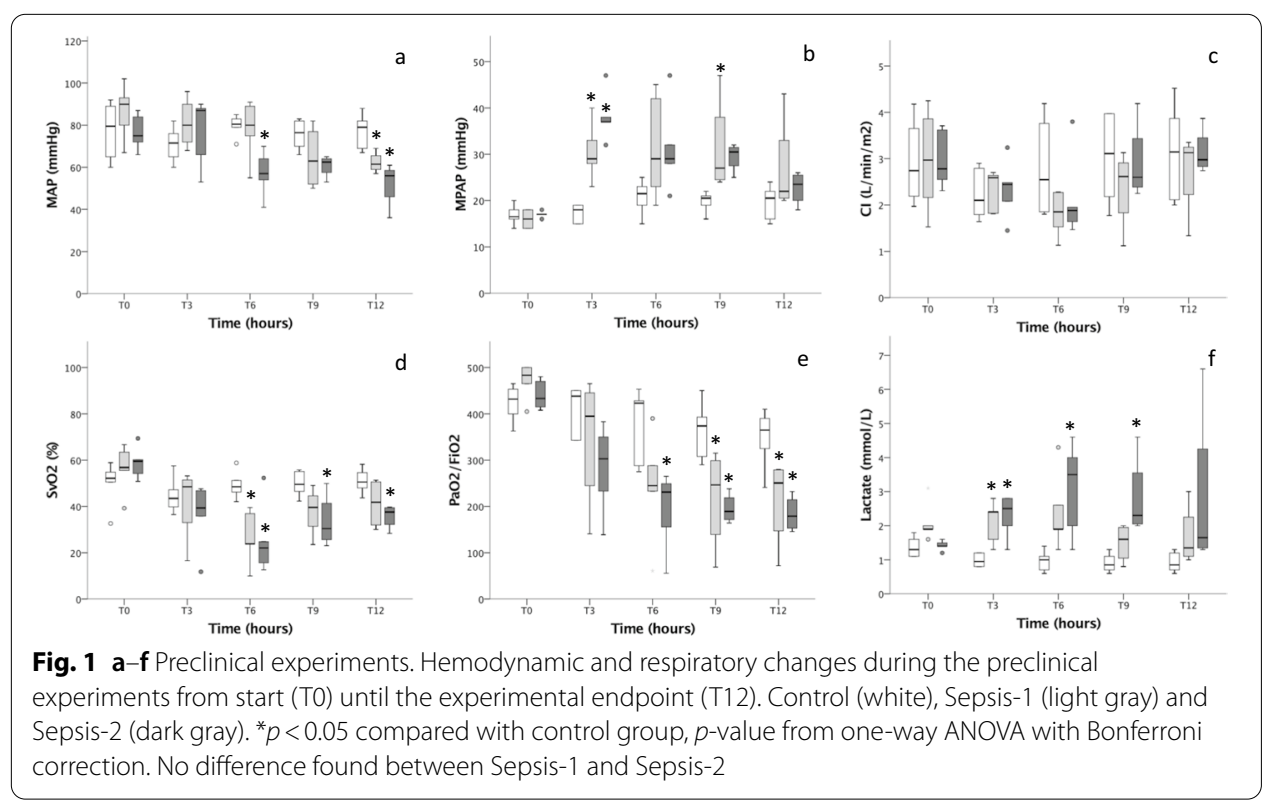



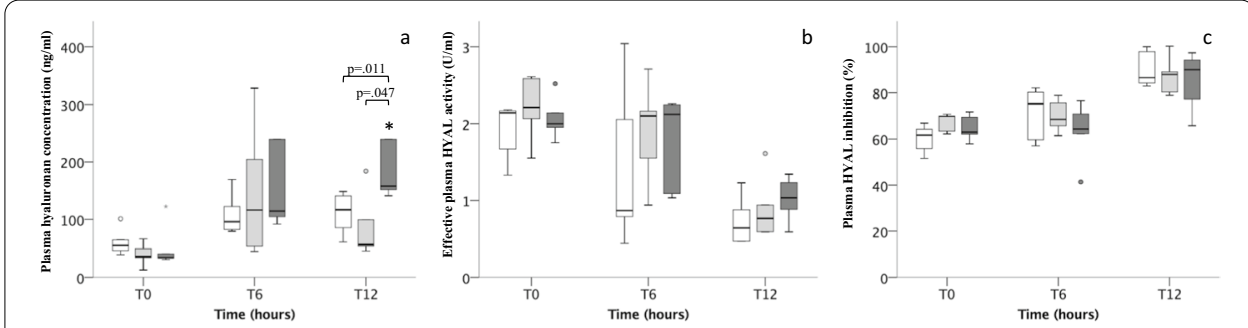

Fig. 2 a-c Preclinical experiments. a Plasma hyaluronan, b effective plasma HYAL activity and $\mathbf{c}$ plasma HYAL inhibition for timepoint T0-6-12. a Plasma hyaluronan concentration increased over time in all groups (Control $p=0.046$, Sepsis-1 and Sepsis-2 $p=0.043$ ). b Effective plasma HYAL activity decreased during the experiment in all groups (Control $p=0.028$, Sepsis- 1 and Sepsis- $p=0.043$ ) whereas $\mathbf{c}$ plasma HYAL inhibition increased significantly in Control and Sepsis-1 over time (Control $p=0.028$, Sepsis-1 $p=0.043$ ). Control (white), Sepsis-1 (light gray) and Sepsis-2 (dark gray). $p$-value from Mann-Whitney $U$ test and Wilcoxon signed-rank test

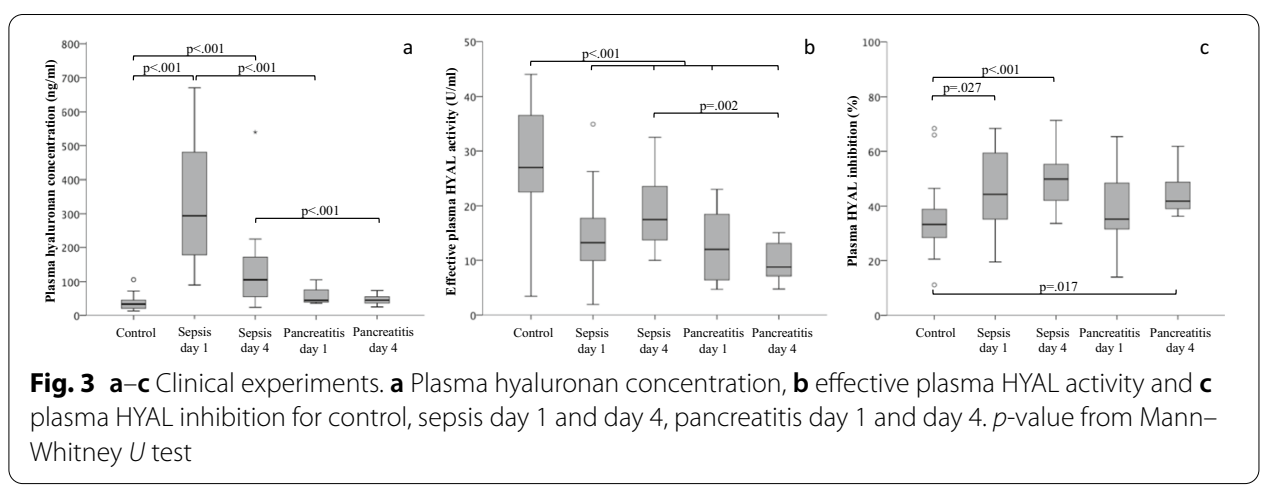

measurements showed a negative correlation between plasma hyaluronan and effective HYAL activity ( $\mathrm{rs}=-0.38, p=0.026)$ and between effective HYAL activity and HYAL inhibition ( $\mathrm{rs}=-0.824, p<0.001$; Additional file 1: Fig. S3a-c). Pooled data showed a negative correlation between effective plasma effective HYAL activity and HYAL inhibition ( $\mathrm{rs}=-0.697, p<0.01$; Additional file 1: Fig. S4a-c) but not between plasma hyaluronan concentration and effective HYAL activity $(p=0.091)$ or plasma hyaluronan concentration and HYAL inhibition $(p=0.501)$.

\section{Septic shock and acute pancreatitis: hyaluronan, effective HYAL activity and HYAL}

\section{inhibition in plasma}

Plasma hyaluronan concentrations in patients with septic shock were higher at day 1 [294 ng/ml (IQR 178-528), $p<0.001$ ] and day 4 [106 ng/ml (IQR 53-179), $p<0.001$ ] compared with healthy volunteers [34 ng/ml (IQR 20-46); Fig. 3]. Plasma hyaluronan concentrations in patients with acute pancreatitis at days 1 and 4 were comparable to that of healthy volunteers ( $p=0.075$ and $p=0.127$, respectively). Plasma hyaluronan concentration was higher for septic shock compared with pancreatitis on both day $1(p<0.001)$ and day $4(p<0.001)$. Effective plasma HYAL activity in healthy volunteers $[26.9 \mathrm{U} / \mathrm{ml}$ (IQR 21.6-36.7)] was higher compared to patients with septic shock at day $1[13.2 \mathrm{U} / \mathrm{ml}$ (IQR 9.2-17.7), $p<0.001]$ and day $4[17.5 \mathrm{U} / \mathrm{ml}(13.6-23.6), \mathrm{p}=0.001]$ and to patients 
with acute pancreatitis at day 1 [12.0 U/ml (IQR 6.4-19.6), $p=0.001]$ and day 4 [8.8 U/ $\mathrm{ml}$ (IQR 6.7-13.6), $p<0.001$ ]. Effective plasma HYAL activity was similar for patients with septic shock and pancreatitis at day $1(p=0.54)$ but not on day $4(p=0.002)$. The endogenous plasma HYAL inhibition in healthy volunteers [33.3\% (IQR 28.3-39.0)] was lower compared to patients with septic shock at day 1 [44.2\% (IQR 33.9-60.0), $p=0.027$ ] and day 4 [49.9\% (IQR 41.2-55.8), $p<0.001]$ and to patients with pancreatitis day at day 4 [41.9\% (IQR 38.3-50.2), $p=0.017$ ] but not day 1 [35.3\% IQR 31.1-51.9), $p=0.542]$. No within-subject correlation between plasma hyaluronan, HYAL and/or HYAL inhibition was found. Pooling data of septic shock patients and healthy volunteers showed a negative correlation between plasma hyaluronan and effective HYAL activity ( $\mathrm{rs}=-0.455$, $p=0.001)$. No correlations were found for acute pancreatitis.

\section{Discussion}

The main finding of the present study is that during experimental 12-h E. coli sepsis, plasma hyaluronan increased with concomitant decrease in effective plasma HYAL activity and increasing endogenous HYAL inhibition. Contrary to our hypothesis, changes of plasma hyaluronan concentration, effective HYAL activity and endogenous HYAL inhibition over time were not specifically associated to $E$. coli sepsis. Rather, general anaesthesia, surgical instrumentation and fluid infusions alone induced comparable effects in the control group. Second, in clinical septic shock of variable microbial origin, high plasma hyaluronan concentration was also associated with lower HYAL activity and higher endogenous HYAL inhibition as compared to healthy volunteers. However, in acute pancreatitis plasma hyaluronan concentration remained low, while effective HYAL activity decreased.

Baseline levels of plasma hyaluronan in the experimental study are consistent with earlier publications [3-6]. Six and twelve hours after starting bacterial infusion the plasma hyaluronan concentration were higher in all groups compared with baseline values. Sterile injury such as surgical instrumentation alone can alter hyaluronan production and metabolism [31]. Furthermore, a rapid infusion of crystalloids similar to the preload bolus in this study showed increased plasma hyaluronan in humans [32]. Finally, preparation was done under clean but non-sterile conditions. Although no positive blood cultures we found in the control group, an alternative source for infection cannot be excluded.

Decreased HYAL activity could increase plasma hyaluronan concentration through different mechanisms. In plasma, circulating HYAL is transported to liver endothelial cells by endocytosis $[34,35]$. Lower HYAL activity resulting in decreased plasma hyaluronan degradation in the liver could contribute to increased plasma hyaluronan. One may also speculate that lower HYAL activity result in accumulation of hyaluronan in the extracellular matrix. Combined with increased hyaluronan production in inflammation $[17,18]$ this could lead to edema formation due to the strong water binding capacity of hyaluronan. Increased lymph flow during sepsis transports the excess of hyaluronan to the lymphatic system [19], where, in normal conditions, most of the hyaluronan is degraded in lymph nodes by HYAL. Decreased HYAL activity in the lymph nodes could result in an increased drainage of hyaluronan into the circulation resulting in increased plasma concentration. 
Effective plasma HYAL activity is decreased in both patients with septic shock and acute pancreatitis, while plasma hyaluronan concentration is only increased in septic shock. This suggests different mechanisms responsible for plasma hyaluronan concentrations in infectious compared to non-infectious inflammation. In addition, increased plasma hyaluronan concentration in septic shock is not caused by decreased HYAL activity alone. Rather, an imbalance between increased production, decreased degradation and altered lymph flow are likely to explain these results.

Increased levels of plasma hyaluronan in sepsis are hypothesized to originate from shedding of the glycocalyx [36] and HYAL has been proposed as a potential important sheddase responsible for damaging the glycocalyx in various clinical conditions [23]. Low effective plasma HYAL activity reported herein makes it unlikely that plasma HYAL contributes to disruption of the glycocalyx in either acute pancreatitis or septic shock. Shedding of the glycocalyx resulting in increased plasma hyaluronan concentrations is observed with several other enzymes [23] and TNF- $\alpha$ [37]. Importantly, our observation is limited to plasma HYAL activity and thereby we cannot exclude any role of plasma membrane associated HYAL. Interestingly, endothelial dysfunction is well described in pancreatitis [38] and if shedding of the glycocalyx is thought to be a source for circulating hyaluronan, an increase of plasma hyaluronan should be expected. However, in our study plasma hyaluronan did not increase in acute pancreatitis. This brings in question if shedding of the glycocalyx significantly contributes to increased plasma hyaluronan.

Septic shock and pancreatitis result in decreased activity of effective HYAL. Surgical instrumentation, fluid infusion and general anaesthesia combined gave similar results. Recent publications on HYAL suggest protective properties in acute inflammation. Exogenous HYAL diminishes the rolling, adhesion and recruitment of neutrophils, decreases cytokine production and limits the albumin diffusion in lung and mesenteric interstitium [39-42]. Furthermore, administration of HYAL has been shown to reduce edema in both pre- and clinical studies [43-45] and is even suggested as a treatment option for COVID-19 [46]. Future research on HYAL and septic shock, acute pancreatitis and major surgery are, therefore, of high interest.

\section{Limitations}

Our study has several limitations. The duration of our 12-h sepsis model might be not long enough to induce plasma hyaluronan concentrations as high as seen in clinical sepsis. Hyaluronan, HYAL activity and HYAL inhibition in plasma were analyzed at two timepoint during ongoing sepsis (T6 and T12). Its is therefor unclear if HYAL activity increases in plasma during early sepsis before HYAL inhibition increases. The small group size in both the preclinical and clinical experiments limit the interpretation of the results. Finally, plasma hyaluronan, HYAL activity and its endogenous inhibition might not accurately reflect any potential changes in the extracellular space or lymphatic system, limiting the generalization of our findings.

\section{Conclusion}

Sepsis but also anaesthesia, surgical procedures and fluid infusions are associated with increasing endogenous plasma HYAL inhibition and decreasing effective plasma HYAL activity, whereas acute pancreatitis is only associated with decreased 
effective plasma HYAL activity. Endogenous plasma HYAL inhibition may regulate plasma HYAL activity in select infections (E. coli) and in inflammation (post-surgery, anaesthesia, fluid infusions). Changes in effective plasma HYAL activity alone do not explain plasma hyaluronan concentrations in bacteremia/sepsis but may, nevertheless, contribute. Increased plasma hyaluronan concentrations in sepsis are most likely caused by increased production or altered lymph flow. Plasma HYAL most likely cannot be considered as an important sheddase.

\begin{abstract}
Abbreviations
ANOVA: Analysis of variance; APACHE II: Acute Physiology and Chronic Health Evaluation; CFU: Colony-forming unit; Cl: Cardiac index; E. coli: Escherichia coli; EDTA: Ethylenediaminetetraacetic acid; ELISA: Enzyme-linked immunoassay; $\mathrm{FiO}_{2}$ : Fractional inspired oxygen; HA: Hyaluronan; HAS: Hyaluronan synthases; HMW-HA: High molecular weight hyaluronan; HYAL: Hyaluronidase; lalp: Inter-alpha-inhibitor protein; MAP: Mean arterial pressure; MPAP: Mean pulmonary arterial pressure; $\mathrm{PaCO}_{2}$ : Arterial partial pressure of carbon dioxide; $\mathrm{PaO}_{2}$ : Arterial partial pressure of oxygen; $\mathrm{PaO}_{2} / \mathrm{FiO}_{2}$ : Arterial partial pressure of oxygen/fractional inspired oxygen; PCWP: Pulmonary capillary wedge pressure; PEEP: Positive endexpiratory pressure; PVRI: Pulmonary vascular resistance index; $\mathrm{SaO}_{2}$ : Arterial oxygen saturation; SIRS: Systemic inflammatory response syndrome; SOFA score: Sequential organ failure assessment score; $\mathrm{SvO}_{2}$ : Mixed venous oxygen saturation; SVRI: Systemic vascular resistance index; TNF-a: Tumor necrosis factor alpha.
\end{abstract}

\title{
Supplementary Information
}

The online version contains supplementary material available at https://doi.org/10.1186/s40635-021-00418-3.

Additional file 1: Figure S1. experiments. Timeline of preclinical exPreclinical perimental design. ABG: arterial blood gas, VBG: mixed venous blood gas. Figure S2a-b. Preclinical experiments. Characteristics of the bacteremia-sepsis model. (a) bacterial infusion in CFU/hour and (b) blood cultures showing CFU per ml blood during bacterial infusion. Control (white; no bacteria infused, no bacteria found in blood cultures), Sepsis-1 (light gray) and Sepsis-2 (dark gray). Figure S3 a-c. Preclinical experiments. (a) Negative within-subject correlation between plasma hyaluronan concentration and effective plasma HYAL activity $(r=-38, p=.026)$ and (b) effective plasma HYAL activity and HYAL inhibition $(r=-.824, p<.001)$ but not for (c) plasma hyaluronan concentration and HYAL inhibition ( $p=.506)$. Each line represents a linear regression between three values $(T=0,6$ and 12) of individual experiments from which a pooled within-subject correlation is calculated according to the Bland Altman method. The experimental groups (control, Sepsis-1 and Sepsis-2) are shown separetely to facilitate the visualization of each individual regression line. Figure S4 a-c. Preclinical experiments. Pooled data of septic shock patients and healthy volunteers showing correlation between (b) effective plasma hyaluronidase activity and hyaluronidase inhibition. No correlation found between (a) plasma hyaluronan concentration and effective hyaluronidase activity and (c) plasma hyaluronan concentration and hyaluronidase inhibition. Correlation and p-value from Spearman's rank-order test.

Additional file 2: Table S1. Preclinical experiments. Experimental interventions for circulatory and respiratory variables, glucose and temperature. Table S2. Preclinical experiments. Baseline conditions, no significant differences between the groups unless for higher lactate in Sepsis-1 compared with Control $(p=.020)$ and Sepsis- $2(p=.039)$. * $p$-value from one-wau ANOVA with Bonferroni correction, $\uparrow p$-value from one-way ANOVA, $\neq p$-value from KruskalWallis test

Additional file 3:Analysis.

Acknowledgements

The authors thank all the staff at the Hedenstierna Laboratory for technical assistance.

\section{Authors' contributions}

$\mathrm{JH}$ and JT conceived the study and designed the experiments with contributions of PS. JH and JT preformed the experimental studies. MS collected the clinical samples. JH preformed both the experimental and clinical laboratory analyses with contributions of $\mathrm{CK}$. JH, MH and JT preformed the statistical analyses. The manuscript was drafted by JH, MH and JT with contributions of PS, MS and EH. All authors have read and approved the final manuscript.

\section{Funding}

Open access funding provided by Uppsala University. This study was supported by ALF-grand from Uppsala University Hospital, Uppsala, Sweden.

\section{Availability of data and materials}

The datasets used and/or analyzed during the current study are available from the corresponding author on reasonable request. 


\section{Declarations}

Ethics approval and consent to participate

Experimental study - The Regional Animals Ethics Committee at the county court of Uppsala approved the experiments (C273/12, date of approval 30 November 2012) and all animals were handled during this study in accordance with the Guide for the Care and Use of Laboratory Animals. Clinical study - The institutional ethics committee of the Helsinki University Central Hospital approved the study (permit number 101/13/03/02/08). Written consent for participation in the study was obtained from either the patient or their next of kin prior to enrolment.

\section{Consent for publication}

Not applicable.

\section{Competing interests}

The authors declare that they have no competing interests.

\section{Author details}

${ }^{1}$ Department of Surgical Sciences, Anesthesiology and Intensive Care, Uppsala University, 75185 Uppsala, Sweden. ${ }^{2}$ Department Medical Biochemistry and Microbiology, Uppsala University, Uppsala, Sweden. ${ }^{3}$ Department of Medical Sciences, Section of Infectious Diseases, Uppsala University, Uppsala, Sweden. ${ }^{4}$ Division of Anaesthesia and Intensive Care Medicine, Intensive Care Units, Department of Surgery, Helsinki University Hospital, Helsinki, Finland. ${ }^{5}$ Department of Medical Cell Biology, Integrative Physiology, Uppsala University, Uppsala, Sweden.

Received: 4 October 2020 Accepted: 23 September 2021

Published online: 11 October 2021

\section{References}

1. Fleischmann C, Scherag A, Adhikari NKJ, Hartog CS, Tsaganos T, Schlattmann P et al (2016) Assessment of global incidence and mortality of hospital-treated sepsis. Current estimates and limitations. Am J Respir Crit Care Med 193:259-272

2. Singer M, Deutschman CS, Seymour CW, Shankar-Hari M, Annane D, Bauer M et al (2016) The third international consensus definitions for sepsis and septic shock (sepsis-3). JAMA 315:801-810

3. Sallisalmi M, Tenhunen J, Kultti A, Tammi M, Pettilä V (2014) Plasma hyaluronan and hemorheology in patients with septic shock: a clinical and experimental study. Clin Hemorheol Microcirc 56:133-144

4. Yagmur E, Koch A, Haumann M, Kramann R, Trautwein C, Tacke F (2012) Hyaluronan serum concentrations are elevated in critically ill patients and associated with disease severity. Clin Biochem 45:82-87

5. Berg S, Brodin B, Hesselvik F, Laurent TC, Maller R (1988) Elevated levels of plasma hyaluronan in septicaemia. Scand J Clin Lab Invest 48:727-732

6. Anand D, Ray S, Srivastava LM, Bhargava S (2016) Evolution of serum hyaluronan and syndecan levels in prognosis of sepsis patients. Clin Biochem 49:768-776

7. Kolar SL, Kyme P, Tseng CW, Soliman A, Kaplan A, Liang J et al (2015) Group B streptococcus evades host immunity by degrading hyaluronan. Cell Host Microbe 18:694-704

8. Stern R, Asari AA, Sugahara KN (2006) Hyaluronan fragments: an information-rich system. Eur J Cell Biol 85:699-715

9. Laurent TC, Fraser JR (1986) The properties and turnover of hyaluronan. Ciba Found Symp 124:9-29

10. Fraser JR, Laurent TC, Laurent UB (1997) Hyaluronan: its nature, distribution, functions and turnover. J Intern Med 242:27-33

11. Itano N, Sawai T, Yoshida M, Lenas P, Yamada Y, Imagawa M et al (1999) Three isoforms of mammalian hyaluronan synthases have distinct enzymatic properties. J Biol Chem 274:25085-25092

12. Brinck J, Heldin P (1999) Expression of recombinant hyaluronan synthase (HAS) isoforms in CHO cells reduces cell migration and cell surface CD44. Exp Cell Res 252:342-351

13. Kolliopoulos C, Bounias D, Bouga H, Kyriakopoulou D, Stavropoulos M, Vynios DH (2013) Hyaluronidases and their inhibitors in the serum of colorectal carcinoma patients. J Pharm Biomed Anal 83:299-304

14. Duran-Reynals $F$ (1933) Studies on a certain spreading factor existing in bacteria and its significance for bacterial invasiveness. J Exp Med 58:161-181

15. Duran-Reynals $F(1939)$ A spreading factor in certain snake venoms and its relation to their mode of action. J Exp Med 69:69-81

16. Mio K, Carrette O, Maibach HI, Stern R (2000) Evidence that the serum inhibitor of hyaluronidase may be a member of the inter-alpha-inhibitor family. J Biol Chem 275:32413-32421

17. Berg S (1997) Hyaluronan turnover in relation to infection and sepsis. J Intern Med 242:73-77

18. Petrey AC, de la Motte CA (2014) Hyaluronan, a crucial regulator of inflammation. Front Immunol 5:101

19. Lebel L, Smith L, Risberg B, Laurent TC, Gerdin B (1989) Increased lymphatic elimination of interstitial hyaluronan during E. coli sepsis in sheep. Am J Physiol 256:H1524-1531

20. Cabrales P, Vázquez BYS, Tsai AG, Intaglietta M (1985) Microvascular and capillary perfusion following glycocalyx degradation. J Appl Physiol Bethesda Md 2007(102):2251-2259

21. Landsverk SA, Tsai AG, Cabrales P, Intaglietta M (2012) Impact of enzymatic degradation of the endothelial glycocalyx on vascular permeability in an awake hamster model. Crit Care Res Pract. 2012:842545

22. Steppan J, Hofer S, Funke B, Brenner T, Henrich M, Martin E et al (2011) Sepsis and major abdominal surgery lead to flaking of the endothelial glycocalix. J Surg Res 165:136-141

23. Becker BF, Jacob M, Leipert S, Salmon AHJ, Chappell D (2015) Degradation of the endothelial glycocalyx in clinical settings: searching for the sheddases. Br J Clin Pharmacol 80:389-402 
24. Skorup P, Maudsdotter L, Lipcsey M, Castegren M, Larsson A, Jonsson A-B et al (2014) Beneficial antimicrobial effect of the addition of an aminoglycoside to a $\beta$-lactam antibiotic in an E. coli porcine intensive care severe sepsis model. PLOS ONE 9:e90441

25. Chain E, Duthie ES (1940) Identity of hyaluronidase and spreading factor. Br J Exp Pathol 21:324-338

26. Hiltunen ELJ, Anttila M, Kultti A, Ropponen K, Penttinen J, Yliskoski M et al (2002) Elevated hyaluronan concentration without hyaluronidase activation in malignant epithelial ovarian tumors. Cancer Res 62:6410-6413

27. Frost Gl, Stern R (1997) A microtiter-based assay for hyaluronidase activity not requiring specialized reagents. Anal Biochem 251:263-269

28. Nawy SS, Csóka AB, Mio K, Stern R (2001) Hyaluronidase activity and hyaluronidase inhibitors. In: lozzo RV (ed) Proteoglycan protoc. Humana Press, Totowa, pp 383-389

29. Stern M, Stern R (1992) An ELISA-like assay for hyaluronidase and hyaluronidase inhibitors. Matrix Stuttg Ger 12:397-403

30. Bland JM, Altman DG (1995) Calculating correlation coefficients with repeated observations: Part 1-correlation within subjects. BMJ 310:446

31. Taylor KR, Yamasaki K, Radek KA, Di Nardo A, Goodarzi H, Golenbock D et al (2007) Recognition of hyaluronan released in sterile injury involves a unique receptor complex dependent on Toll-like receptor 4, CD44, and MD-2. J Biol Chem 282:18265-18275

32. Berg S, Engman A, Hesselvik JF, Laurent TC (1994) Crystalloid infusion increases plasma hyaluronan. Crit Care Med 22:1563-1567

33. Toida T, Ogita Y, Suzuki A, Toyoda H, Imanari T (1999) Inhibition of hyaluronidase by fully O-sulfonated glycosaminoglycans. Arch Biochem Biophys 370:176-182

34. Harada H, Takahashi M (2007) CD44-dependent intracellular and extracellular catabolism of hyaluronic acid by hyaluronidase-1 and -2. J Biol Chem 282:5597-5607

35. Gasingirwa M-C, Thirion J, Mertens-Strijthagen J, Wattiaux-De Coninck S, Flamion B, Wattiaux R et al (2010) Endocytosis of hyaluronidase-1 by the liver. Biochem J 430:305-313

36. Köhler M, Kaufmann I, Briegel J, Jacob M, Goeschl J, Rachinger W et al (2011) The endothelial glycocalyx degenerates with increasing sepsis severity. Crit Care 15:P22

37. Henry CB, Duling BR (2000) TNF-alpha increases entry of macromolecules into luminal endothelial cell glycocalyx. Am J Physiol Heart Circ Physiol 279:H2815-2823

38. Dumnicka P, Maduzia D, Ceranowicz P, Olszanecki R, Drożdż R, Kuśnierz-Cabala B (2017) The interplay between inflammation, coagulation and endothelial injury in the early phase of acute pancreatitis: clinical implications. Int J Mol Sci 18:1

39. Huang Z, Zhao C, Chen Y, Cowell JA, Wei G, Kultti A et al (1950) Recombinant human hyaluronidase PH20 does not stimulate an acute inflammatory response and inhibits lipopolysaccharide-induced neutrophil recruitment in the air pouch model of inflammation. J Immunol Baltim Md 2014(192):5285-5295

40. Fronza M, Muhr C, da Silveira DSC, Sorgi CA, Rodrigues SFP, Farsky SHP et al (2016) Hyaluronidase decreases neutrophils infiltration to the inflammatory site. Inflamm Res 65:533-542

41. Qiu XL, Brown LV, Parameswaran S, Marek VW, Ibbott GS, Lai-Fook SJ (1999) Effect of hyaluronidase on albumin diffusion in lung interstitium. Lung 177:273-288

42. Parameswaran S, Brown LV, Ibbott GS, Lai-Fook SJ (1994) Effect of concentration and hyaluronidase on albumin diffusion across rabbit mesentery. Microcirc N Y N 1999(6):117-126

43. Waldenström A, Martinussen HJ, Gerdin B, Hällgren R (1991) Accumulation of hyaluronan and tissue edema in experimental myocardial infarction. J Clin Invest 88:1622-1628

44. Johnsson C, Hällgren R, Elvin A, Gerdin B, Tufveson G (1999) Hyaluronidase ameliorates rejection-induced edema. Transpl Int 12:235-243

45. Venkatesh KH, Rao GSU (2007) Treatment of supraglottic airway edema by local hyaluronidase. Anesthesiology 106:1254-1255

46. Shi Y, Wang Y, Shao C, Huang J, Gan J, Huang X et al (2020) COVID-19 infection: the perspectives on immune responses. Cell Death Differ 27:1451-1454

\section{Publisher's Note}

Springer Nature remains neutral with regard to jurisdictional claims in published maps and institutional affiliations. 